\title{
VARIATION IN CHIASMA FREQUENCY IN CEPAEA NEMORALIS
}

\author{
DAVID J. PRICE \\ Department of Biology and Geology, The Polytechnic of North London, \\ Holloway, London N7 8DB
}

Received 22.iii.73

\section{SUMMARY}

\begin{abstract}
Nineteen populations of the snail Cepaea nemoralis were sampled from a geographically restricted area and analysed for variation in chiasma frequency. Most variation occurs in the single A group bivalent which can have up to six chiasmata. This is higher than has previously been described. Chiasmata are not apparently localised except that when only one is present it is almost always central in position. Results suggest that interstitial chiasmata and terminal chiasmata may be under different genetic controls. Increased chiasma frequency on the A group bivalent is associated with increased between cell variance. It is suggested that this may be a means of compensating for the lack of release of genetic variability due to recombination by the other twenty-one bivalents which are of small size and nearly always have one chiasma. Significant differences between populations in both chiasma frequency and between cell variance were found; a limited amount of data suggests that these differences appear to be stable between successive years.
\end{abstract}

\section{INTRODUCTION}

Ат least five strongly linked loci are involved in the shell colour and banding polymorphism of the snail Cepaea nemoralis. Most populations are polymorphic and may show linkage disequilibrium (see reviews by Ford, 1971; Williamson, 1972). Bantock (1972) examined chiasma frequency in one highly polymorphic population showing pronounced linkage disequilibrium and found that chiasmata were limited in number and apparently localised in position. He suggested that both the localised chiasmata and the strong linkage between the shell colour and banding loci may be a reflection of the need to preserve epistatic gene complexes in this population and also that populations with a more restricted range of phenotypic variation may show a greater diversity of chiasma frequency and position.

The purpose of this paper is to examine the extent of chiasma frequency variation in a number of populations of $C$. nemoralis showing different polymorphic states.

\section{Materials AND methods}

Adult snails were collected from 19 colonies between 6th and 9th April 1972. Each sample was collected from an area of no more than 20 metres radius or 30 metres extent when linear; the composition and location of population samples is given in table 1 . The limited range of shell variation in the area is reflected in the table by the high frequency of pink mid-banded snails and the low frequency of yellow shelled snails. The animals were kept 
under outdoor conditions in plastic boxes containing damp filter paper and natural chalk. They were fed on carrot.

In order to eliminate any bias due to seasonal variation which might occur if all the snails from one population were prepared before those from another, one snail from each population was prepared contemporaneously. Heatdried films of the ovotestis were prepared (by a method previously described; Bantock and Price, in press) from between 11 and 24 snails from each sample.

TABLE 1

Location and composition of the population samples

Shell colour and banding morph

$\begin{array}{cc}\text { Population } & \text { Grid reference } \\ 1 & \text { STO3.054.374 } \\ 2 & \text { STO3.055.398 } \\ 3 & \text { STO4.009.426 } \\ 4 & \text { STO3.052.375 } \\ 5 & \text { STO4.017.407 } \\ & \\ 6 & \text { STO4.017.401 } \\ 7 & \text { STO4.017.425 } \\ 8 & \text { STO3.037.376 } \\ 9 & \text { STO4.033.403 } \\ 10 & \text { STO4.009.433 } \\ 11 & \text { STO4.018.425 } \\ 12 & \text { STO3.062.374 } \\ 13 & \text { STO3.066.373 } \\ 14 & \text { STO3.055.399 } \\ 15 & \text { STO4.067.404 } \\ 16 & \text { STO3.025.302 } \\ 17 & \text { STO4.031.423 } \\ 18 & \text { STO4.032.420 } \\ 19 & \text { STO4.033.418 }\end{array}$

\begin{tabular}{|c|c|c|c|c|c|c|c|c|c|c|}
\hline B0 & P0 & Yo & B3 & P3 & Y3 & B5 & P5 & Y5 & Others & Total \\
\hline - & 3 & - & - & 15 & - & - & - & - & - & 18 \\
\hline - & 2 & - & - & 9 & - & - & - & - & 5 P3 spd. & 16 \\
\hline - & 3 & - & - & 16 & - & - & 12 & 1 & - & 32 \\
\hline 1 & 15 & - & - & 41 & - & - & - & - & - & 57 \\
\hline 1 & - & - & - & 63 & 15 & - & 2 & - & $\begin{array}{l}1 \text { B3 spd., } 1 \text { P3 spd., } \\
2 \text { Y3 spd. }\end{array}$ & 85 \\
\hline - & - & - & - & 22 & 8 & - & - & - & 1 P3 spd. & 31 \\
\hline - & - & - & - & 24 & 6 & - & 20 & 20 & - & 70 \\
\hline 6 & 21 & - & - & 27 & - & - & - & - & - & 54 \\
\hline - & 17 & 1 & - & 9 & - & - & - & - & - & 27 \\
\hline 7 & 4 & - & - & 16 & - & - & 24 & - & - & 51 \\
\hline 2 & - & - & - & 23 & 7 & - & 27 & 7 & - & 66 \\
\hline- & 5 & - & - & 13 & - & - & - & - & - & 18 \\
\hline 6 & 4 & - & - & 17 & - & - & - & - & - & 27 \\
\hline - & 3 & - & - & 5 & - & - & - & - & 6 P3 spd., 1 Y3 spd. & 15 \\
\hline 8 & 1 & - & - & 13 & - & - & 1 & - & - & 23 \\
\hline 4 & - & - & 一 & 17 & - & - & 7 & - & - & 28 \\
\hline 13 & 10 & - & - & 17 & - & - & 10 & - & - & 50 \\
\hline 28 & 22 & - & - & 14 & - & - & 1 & - & - & 65 \\
\hline 7 & 10 & - & 1 & 22 & - & 2 & 11 & - & - & 53 \\
\hline
\end{tabular}

The chromosome complement of $C$. nemoralis has been divided into three groups (Bantock, loc. cit.). There is a large metacentric chromosome about $7 \mu \mathrm{m}$ long comprising the A group, a smaller metacentric forming the $\mathrm{B}$ group, and 20 pairs of smaller chromosomes which form the $\mathrm{C}$ group. During diplotene, B and C group bivalents were considered together for analysis. Chiasmata on the A group bivalent were scored separately.

A preliminary investigation showed that there was no reduction in the frequency of chiasmata between early diplotene and early diakinesis $(P \gg 0.05)$, nor was there any increase in the terminalisation coefficient $(\mathrm{P} \gg 0.05)$. However, due to progressive contraction, it becomes difficult to record both frequency and positions of chiasmata in diakinetic cells so that only diplotene cells were scored. The first 30 suitable cells were used from each animal; in all a total of 10,590 cells has been examined.

Although, it is not possible to record the positions of chiasmata accurately in relation to the centromere in Cepaea, the approximate positions of chiasmata were recorded. In view of the localisation which Bantock (loc. cit.) found, he classified chiasmata as either proximal or proterminal. Zarchi et al. (1972) produced evidence that in wheat terminal chiasmata were under a different genetic control than the remaining chiasmata. In view of this and 


\section{Plate I}

Spermatocytes of $C$. nemoralis in diplotene. $\quad \times 1250$

The comments refer to the single A group bivalent (arrowed in 1)

1. One chiasma, central in position.

2. A single, non-central chiasma.

3. Two interstitial chiasmata, but without a central chiasma.

4. Two chiasmata; one a terminal, the other a central interstitial chiasma.

5. Two interstitial chiasmata, one central in position.

6. Three interstitial chiasmata; although two chiasmata occur very close to the ends of the bivalent these are not classified as terminals.

7 and 8 . Both cells have four chiasmata; note the different positions of chiasmata in the two cells.

9. Five chiasmata, all interstitial.

10. Six chiasmata; one terminal in position.

All the B and C group bivalents have a single chiasma except in cells 6 and 10 where one bivalent in each cell has two chiasmata (arrowed). 


$$
\int_{1}^{-2}
$$

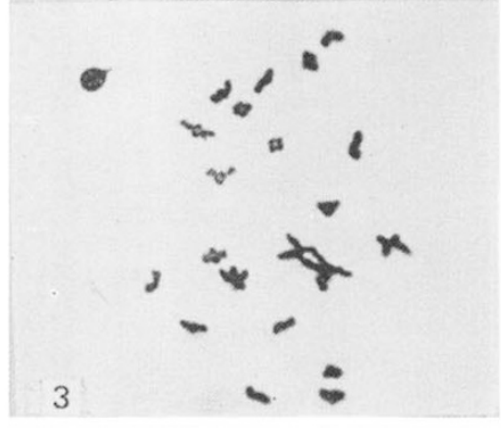

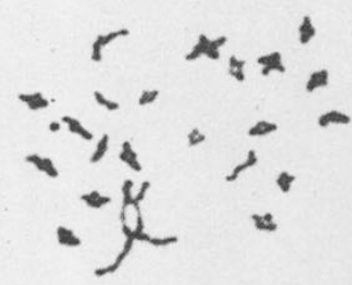

5

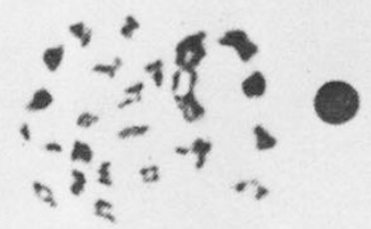

$$
7
$$

$$
9 a^{2+2}
$$

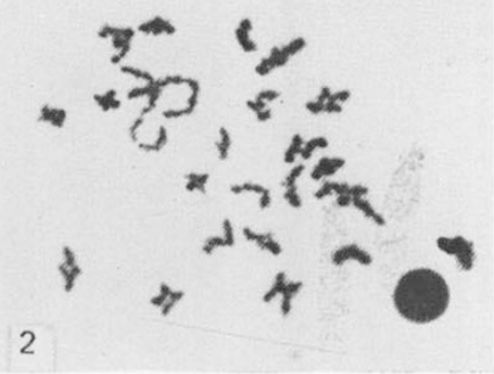

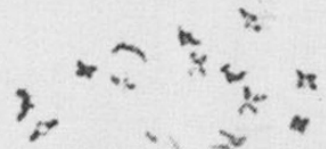

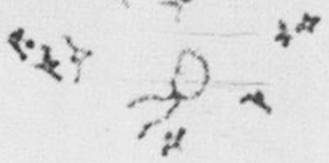

4

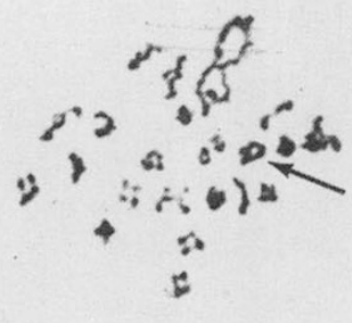

6

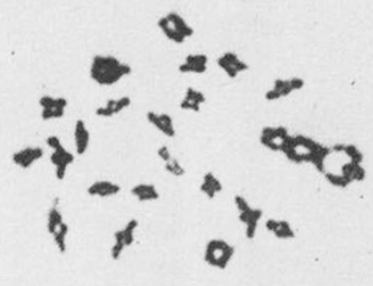

8

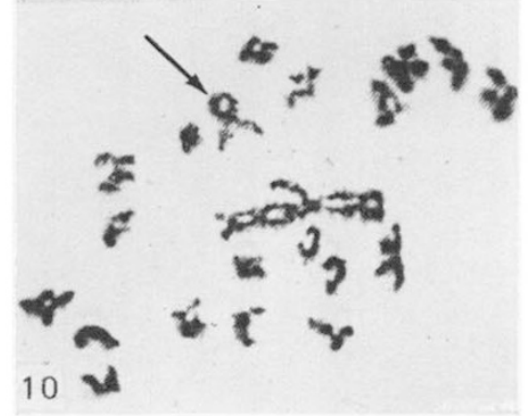


of the range of variation in chiasma position encountered in the present study, chiasmata were classified as interstitial or terminal, terminal chiasmata being those beyond which chromosome arms could not be seen.

\section{Results}

\section{(i) Methodology}

The major variation in chiasma frequency occurs on the A group bivalent alone; most of the analysis is therefore devoted to this bivalent alone. On this bivalent for each individual, the $m$ ean number of total chiasmata, the mean number of interstitial chiasmata, and the mean number of terminal chiasmata were calculated. For each of these statistics, the variance between the 30 cells of an individual was calculated and transformed to allow statistical analysis. The transformation used was $\log _{10}$ $\left(S^{2}+1 \cdot 0\right)$, where $S^{2}$ is the between-cell variance. On the B and $\mathrm{C}$ group bivalents, only the numbers of chiasmata were recorded.

The raw data are extensive but may be obtained on request from the author.

\section{(ii) Chiasma position}

Plate I shows that chiasmata may occur along most of the length of the A bivalent and overall are not localised. However, a chiasma in approximately the middle of the bivalent, and hence presumably close to the centromere, occurs in 97 per cent of the cells (plate I, I, 3, 5 and 6). Furthermore, in 3030 cells having one chiasma on the A bivalent, the chiasma is central in all but two. Plate I, 2 shows one of these exceptions. A cell with more than one chiasma but without the central one is seen in plate I, 3. Differences in position among cells with the same chiasma frequency can be seen in plate I, 3-5 and plate I, 7 and 8 . Plate I, 4 shows clearly a terminal chiasma.

\section{(iii) Chiasma frequency}

Of 10,590 cells examined, 98 per cent had three or less chiasmata on the A group bivalent. Cells with higher numbers are seen in plate I, 7-10. The population mean frequency for total chiasmata on the A bivalent varies between 1.66 and $2 \cdot 25$. This is much higher than the average frequency of 1.27 found by Bantock (loc. cit.). Interstitial chiasmata means vary between 1.29 and 1.91. The highest population mean for terminal chiasmata is 0.53 ; since the maximum possible is $2 \cdot 0$, this figure indicates that only 25 per cent of the potential sites for terminal chiasmata are occupied in this population. Interpopulational variation in total chiasma frequency is significant $(P<0.001)$. Separate analyses for interstitial and terminal chiasmata show similar significant differences although the variance ratio for interstitial chiasmata is increased whereas that for terminal chiasmata is decreased.

Examination of plate I shows that most of the B and $\mathrm{G}$ group bivalents have only one chiasma. Occasionally two are present as in plate I, 6 and 10 . Population 3 had 45 bivalents with two chiasmata out of a total of 13,860 $\mathrm{B}$ and $\mathrm{C}$ group bivalents. This is the highest frequency which has been encountered. 


\section{(iv) Variance between cells}

The between-cell variance is a measure of the uniformity of chiasma formation within that individual. Animals with a high variance will produce a more diverse array of gametes than will animals with a low variance. Population means for between-cell variance differ and an analysis of variance showed that the differences between populations are significant $(P<0.001)$. The difference is most pronounced in the interstitial chiasmata.

\section{(v) Stability of chiasma frequency}

In view of the highly significant differences between populations it is obviously desirable to know how stable chiasma frequency is. The population described by Bantock (loc. cit.) from Haddon Hill was resampled in 1972 and compared with the results of the previous year. Table 2 shows that in this population at least both chiasma frequency and within individual variance have remained similar between successive years.

TABLE 2

A comparison of mean chiasma frequencies and between cell variances between successive years in the Haddon Hill population

\begin{tabular}{cccccccc} 
& \multicolumn{2}{c}{ Chiasma frequency } & & \multicolumn{3}{c}{ Between-cell variances } \\
\cline { 2 - 5 } 1971* & 1.27 & 1.07 & 0.20 & & 0.0735 & 0.0272 & 0.0643 \\
1972 & 1.39 & 1.06 & 0.33 & & 0.0987 & 0.0235 & 0.0808 \\
P ("t " test) & $0.4-0.5$ & $>0.5$ & $0.2-0.4$ & & $0.2-0.4$ & $>0.5$ & $0.2-0.4$
\end{tabular}

* 1971 data rearranged from Bantock (1972).

(vi) Correlations between characteristics

Interstitial chiasma frequency correlates strongly with total chiasma frequency, as does terminal chiasma frequency. However, there is little correlation between interstitial and terminal chiasma frequencies. There is a tendency for interstitial between-cell variance to show a positive correlation with the total variance although five populations do not. Terminal variance, however, usually does not show this correlation, although again there are exceptions, and terminal variance shows little evidence of a correlation with interstitial variance. Nearly all populations show strong correlations between interstitial chiasma frequency and variance, and between terminal chiasma frequency and variance. However, the correlation between total chiasma frequency and total between-cell variance is less marked and is absent from more than half the populations.

Thus, as both interstitial and terminal chiasma frequency increase so does the appropriate variance. However, the results (table 3) suggest that interstitial and terminal chiasmata are to a large extent independent of each other.

\section{Discussion}

The results indicate that variation in numbers and position of chiasmata in spermatocytes of Cepaea is greater than has previously been shown. Most $\mathrm{B}$ and $\mathrm{C}$ group bivalents have only one chiasma which they presumably need to ensure the orderly segregation of homologous chromosomes later in 


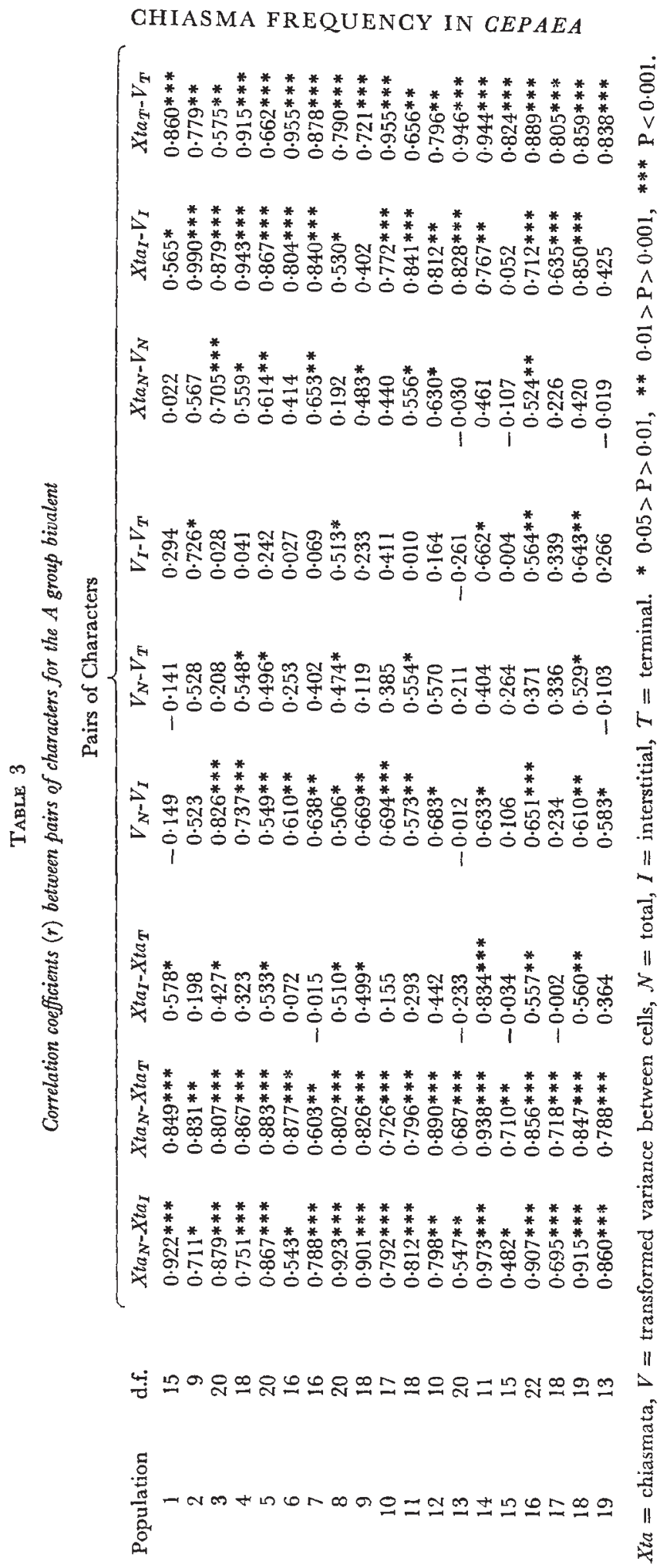


meiosis. More interesting are the observations concerning the single A group bivalents. Analyses of variance show that there are large differences between populations for interstitial and terminal chiasma frequencies and for betweencell variances.

Terminal chiasmata, which represent events at or near the ends of bivalents, are presumably not as important as interstitial chiasmata in producing effective recombination between loci. Terminal chiasmata could represent earlier recombination events which occurred elsewhere along the bivalent and subsequently moved to a terminal position, but terminalisation does not appear to occur in Cepaea. Zarchi et al. (loc. cit.) suggested that the role of terminals in wheat may be to ensure proper segregation at anaphase $I$. However, this seems unlikely in Cepaea for two reasons. Firstly, the frequency of terminal chiasmata is too low for them to have this role, and secondly, it seems probable that this function is performed by the central chiasma which is present in almost all the A group bivalents with only one chiasma. The presence of $\mathrm{B}$ chromosomes in wheat reduces the number of chiasmata in the normal chromosome complement (Zarchi et al., loc. cit.). This effect is confined to the terminal chiasmata (the frequency of interstitial chiasmata remaining the same or even being slightly increased). These authors suggested that terminal chiasmata may be under a different genetic control. The present results suggest that this may also be the case in Cepaea. For example, in most populations interstitial chiasma frequency does not correlate with terminal chiasma frequency. Similarly, there is little or no correlation between the interstitial cell variance and the terminal cell variance.

Interstitial chiasmata are presumably important in recombining lengths of homologous chromosomes, and if gene loci are heterozygous they lead to effective genetic recombination. In Cepaea, variation in chiasma frequency is very largely due to variation in the A group bivalent. It is possible that mechanical considerations due to the small size of the $B$ and $C$ group bivalents limit the formation of extra chiasmata on these bivalents. Individuals with a high chiasma frequency and a high cell variance on the A group bivalent may form a more diverse array of gametes than snails with the same chiasma frequency but a low variance. Hence the occurrence of a high cell variance may be a means of increasing the effective recombination in a population over that which can be obtained merely by a high chiasma frequency and low cell variance. This could compensate for the lack of release of variability by the $\mathbf{B}$ and $\mathbf{C}$ group bivalents.

It was suggested by Bantock (loc. cit.) that reduced variability at the colour and banding loci may be associated with increased chiasma frequencies. I am grateful to Dr Bantock for providing me with the following information concerning variation in morph frequency in $C$. nemoralis in the area from which samples in the present study were taken. Of 280 samples of 21 or more animals, 130 are monomorphic for dark pink; only 43 samples contain yellows and in only one sample (population 7 in this paper) does the frequency of this colour rise above 25 per cent. There is a pronounced negative association between the distribution of yellow and banding so that populations polymorphic $\left(C^{p}\right.$ and $\left.C^{y}\right)$ at the $\mathrm{C}$ locus are monomorphic $\left(B^{b}\right)$ at the $B$ locus. There is one exception to this, population 9 in this paper. Populations without yellows contain both bandeds and unbandeds. Overall it is impossible to detect linkage between the colour and banding loci in the area and the distributional data suggest that there is selection against the 
simultaneous occurrence of $C^{y}$ and $B^{o}$. This contrasts with the Haddon Hill population described by Bantock (loc. cit.) which is polymorphic at 3 loci and where the disequilibrium suggests strong linkage between $C^{y}, B^{o}$ and $P^{n}$.

In view of this it is interesting to note that all the populations in this paper have higher chiasma frequencies than the Haddon Hill population. Two populations (17 and 18) may show linkage disequilibrium in that they lack brown banded individuals. However, Cain et al. (1960) showed that there may be an epistatic effect of brown on the expression of banding so that genetically brown banded individuals appear phenotypically unbanded. These two populations do not, however, have a low chiasma frequency; nor does there appear to be, in this area at least, any obvious correlation between chiasma frequency and the number of shell morphs in a population.

In the present study, however, there is no obvious correlation between chiasma frequency and physical features such as altitude. However, there is an indication that chiasma frequency (and particularly interstitial chiasma frequency) increases with apparent decreasing population density. Cepaea has a restricted mobility and this, coupled with a population of small size, will increase the level of inbreeding. Higher chiasma frequencies in small populations would compensate for this.

Acknowledgments.-I would like to thank Dr C. R. Bantock for suggesting the field of study and for reading the manuscript. I would also like to thank him and Miss K. Noble of the Leonard Wills Field Centre for help in the collection of materials. This work was carried out during the tenure of a research assistantship.

\section{REFERENCES}

BANTOCK, C. R. 1972. Localisation of chiasmata in Cepaea nemoralis L. Heredity, 29, $213-221$. BANTOCK, C. R., AND PRICE, D. J. 1973. The cytology of Cepaea nemoralis. In Laboratory Manual of Cell Biology (ed. D. O. Hall et al.). English Universities Press, London. (In Press.) CAIN, A. J., KING, J. M. B., AND SHEPPARD, P. M. 1960. New data on the genetics of polymorphism in the snail Cepaea nemoralis. Genetics, 45, 393-411.

FORD, E. B. 1971. Ecological Genetics, 3rd ed. Chapman and Hall, London.

williamson, M. 1972. The Analysis of Biological Populations. Edward Arnold, London.

ZARCHI, Y., SIMGHEN, G., HILLEL, J., AND SGHAAP, T. 1972. Chiasmata and the breeding system in wild populations of diploid wheats. Chromosoma, 38, 77-94. 